\title{
Three-Dimensional Time-of-Flight Secondary Ion Mass Spectrometry and DualBeam FIB/SEM Imaging of Lithium-ion Battery Cathode
}

\author{
Chengge Jiao ${ }^{1 *}$, Lex Pillatsch ${ }^{2,3}$, Johannes Mulders ${ }^{1}$ and David Wall ${ }^{1}$ \\ 1. ThermoFisher Scientific, Eindhoven, the Netherlands. \\ 2. Empa (Swiss Federal Laboratories for Materials Science and Technology), Laboratory for Mechanics \\ of Materials and Nanostructures, Thun, Switzerland. \\ 3. TOFWERK AG, Thun, Switzerland. \\ * Corresponding author: chengge.jiao@thermofisher.com
}

Lithium ion batteries (LIBs) cathode analysis plays a critical role for determining battery properties such as capacity, voltage and energy density. Since lithium ions move from the negative electrode to the positive electrode during discharge and back when charging, battery performance is determined by the binding between lithium ions and the electrode material. Lithium ion batteries operates longer and faster when their electrodes are treated with hydrogen [1].

Energy dispersive x-ray (EDS) with a dual beam FIB/SEM microscope is a standard technique, which can provide Li-ion battery chemical analysis. However, detection of hydrogen by EDS is impossible and detection of lithium requires well defined conditions and is very challenge [2]. Secondary Ion Mass Spectrometry (SIMS) is an materials chemical analysis technique based on collection and separation of isotopes according to their mass-to-charge ratio $(\mathrm{m} / \mathrm{z})$ from detected secondary ions. The secondary ions are emitted from solid material surfaces as a small fraction of charged particles among in sputtering particles by primary ion beam bombardment.

SIMS as an add-on technique to a dual beam FIB/SEM microscope allows focused ion beam used as an analytical beam. The ion beam interaction volume for most materials is a few tens of nanometers, which is orders of magnitude smaller than that generated by an electron beam at the same energy. Therefore, added on a SIMS to a dual beam can achieve a better lateral $(\mathrm{x}, \mathrm{y})$ and depth (z) resolution for chemical analysis comparing to a common SEM/EDS technique. TOFWERK TOF-SIMS analyzer [3] uses an orthogonal extraction design to pulse secondary ion beam. This allows for continues sputtering of the sample surface with the primary FIB beam. The collection optics is retractable and SIMS data collection is from the sample at eucentric height for a dual beam FIB/SEM microscope. TOF SIMS lateral resolution is approximately $40 \mathrm{~nm}$ to $50 \mathrm{~nm}$ for focused gallium ion beam. Positive and negative secondary ions can be detected. The primary ion beam is raster over the sample surface and a full mass spectrum is recorded for every scanned pixel. Ion images are reconstructed by showing the intensity distribution of the specific isotope.

In this paper, we first demonstrate light element detection by add-on TOF SIMS with a dual beam FIB/SEM microscope (as shown in the Figure 1). The intensity distribution of light elements, such as hydrogen $(\mathrm{H})$, lithium $(\mathrm{Li})$, fluoride $(\mathrm{F})$, oxygen $(\mathrm{O})$ was recorded for FIB milled cross-sections in a $\mathrm{LiNi}_{\mathrm{x}} \mathrm{Mn}_{\mathrm{y}} \mathrm{Co}_{1-\mathrm{x}-\mathrm{y}} \mathrm{O}_{2}$ (NMC) battery cathode sample. Then, we describe 3D SIMS data acquisitions and present 3D reconstructed lithium, fluoride, carbon and oxygen element distributions in Li-ion battery NMC cathode. Two methods have been used to acquire 3D SIMS data from Li-ion cathode by a dual beam/TOF SIMS system, 1) SIMS depth profiles data was acquired followed by 3D reconstruction; 2) FIB mill serial section for 2D SIMS data acquisition and subsequent 3D reconstruction. A 3D 
reconstructed lithium distribution result is illustrated in the Figure 2. We compared the difference between two methods for 3D-SIMS imaging in the paper.

In conclusion, TOF SIMS add-on to the dual beam SEM/FIB system allows ion beam to be used not only for milling and deposition but also as a beam for chemical analysis. Light mass detection capability and the good detection efficiencies for lithium metal oxides materials makes the SIMS technique suitable for 2D/3D TOF SIMS work for Li-ion battery research.

\section{References:}

[1] J Ye et al., Scientific Reports [Online] 5, 16190, (2015), https://www.nature.com.

[2] P Hovington et al., Scanning 38, (2016), p.571-578.

[3] L Pillatsch, F Ostlund, J Michler, Progress in Crystal Growth and Characterization of Materials [Online] December 2018, https://doi.org/10.1016/j.pcrysgrow.2018.10.001.
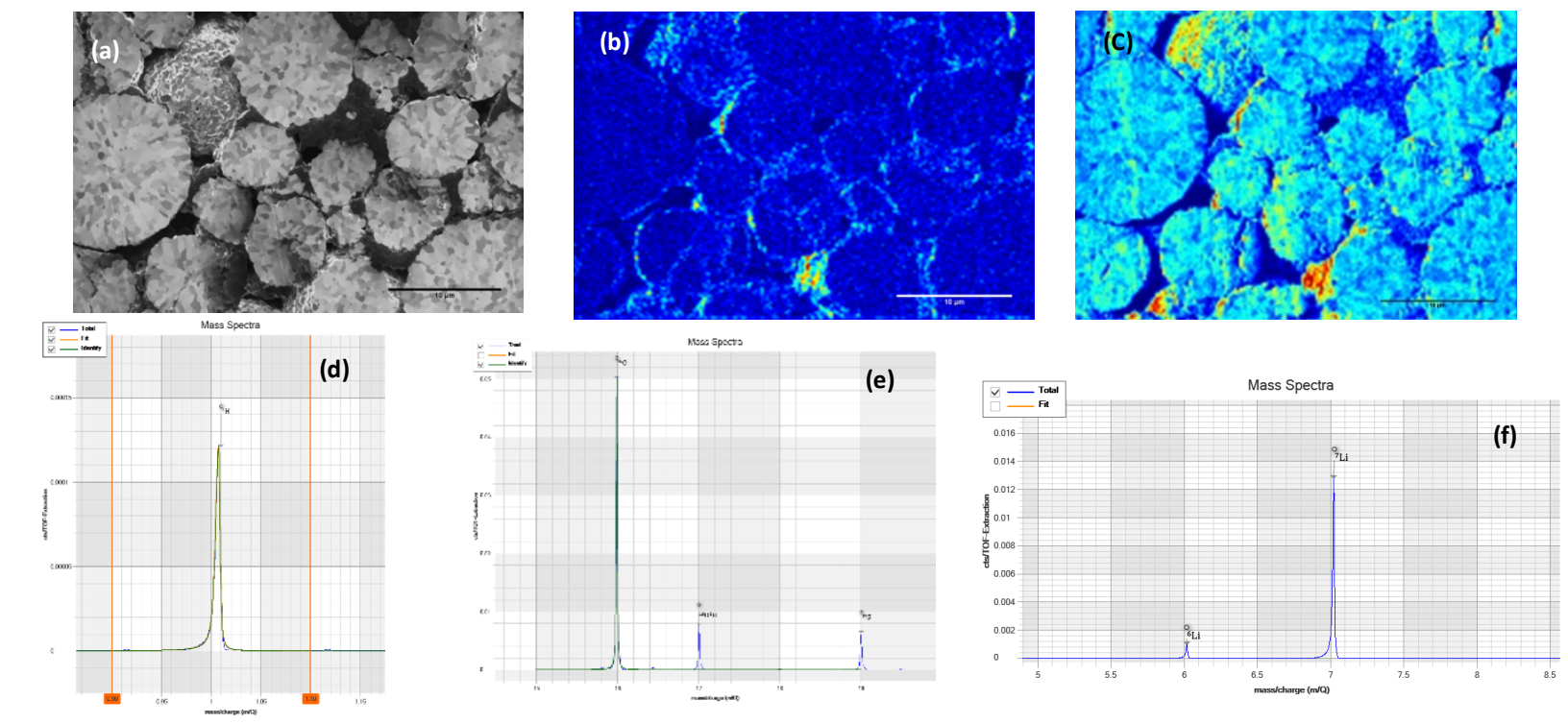

Figure 1. Hydrogen and lithium detections from $\mathrm{LiNiCoMnO}$ cathode cross-section prepared by FIB mill. (a) FIB induced secondary electron image acquired with beam current of $430 \mathrm{pA}$ at $30 \mathrm{kV}$; (b) ${ }^{1} \mathrm{H}^{-} \mathrm{TOF}$ SIMS image illustrates hydrogen distribution; (c) ${ }^{7} \mathrm{Li}^{+}$SIMS image; (d) and (e) Mass spectra of ${ }^{1} \mathrm{H}^{-}$, ${ }^{16} \mathrm{O}^{-}$, $\left[{ }^{16} \mathrm{O}^{1} \mathrm{H}\right]^{-}$and ${ }^{19} \mathrm{~F}^{-}$collected with a negative ion extraction; (f) Mass spectra of ${ }^{6} \mathrm{Li}^{+}$and ${ }^{7} \mathrm{Li}^{+}$collected with a positive ion extraction.


Figure 2. Reconstructed TOF SIMS 3D images illustrating the ${ }^{7} \mathrm{Li}^{+}$distribution in $3 \mathrm{D}$. (a) 3D data was acquired by serial FIB sections; (b) 3D data was acquired based on conventional SIMS depth profile. 